\title{
Approximation of the growth and survival curve of a non-subcultured cell culture within the framework of the stationary phase aging model
}

\author{
A.A. Klebanov, G.V. Morgunova, A.N. Khokhlov* \\ School of Biology, Lomonosov Moscow State University, Moscow, Russia \\ *e-mail:khokhlov@mail.bio.msu.ru
}

Key words: stationary phase aging, cell cultures, growth curve, survival curve, approximation

Motivation and Aim: The most reliable way to test geroprotectors is to construct the survival curves of organisms. For a long time the phenomenon of our stationary phase aging model was considered as accumulation in cultured cells, the proliferation of which is restricted by some way (for example, contact inhibition), of "age-related" lesions similar to age-related changes in vivo. At the same time, in our cell-kinetic model, only the growth rate of the culture and the saturating density reached, which characterizes the "biological age" of the cells, were estimated, and the kinetics of their death was not considered. However, we subsequently showed that during "stationary phase aging" the cells, accumulating "age-related" damage, die out in accordance with the Gompertz equation, i. e. really grow old, and the probability of their death increases with time exponentially, as in aging animals/humans. Thus, the growth and death curves of a non-subcultured cell culture could be approximated by two different equations. At the same time, a number of data showed that both parts of the curve can be interrelated. We hypothesized that the creation of a single equation describing both the initial growth of the culture and the death of cells in the stationary phase could significantly improve the efficiency of testing various geroprotectors and geropromoters on this model system. Methods and Algorithms: The corresponding growth and death curves of the nonsubcultured cell culture were obtained using transformed Chinese hamster cells of the B11-dii FAF28 line which were incubated at $37{ }^{\circ} \mathrm{C}$ in Dulbecco's modified Eagle's medium supplemented with $10 \%$ bovine serum in hermetically sealed vials for 40 55 days. For subsequent mathematical processing, new data on the influence of the buffer capacity of the growth medium and exogenous 8-oxo-2'-deoxoguanosine on the growth and stationary phase aging of cells were used as well as earlier results concerning the effects of $\mathrm{C}_{60}$-fullerene and Quinton Marine Plasma in this model system. Approximation was carried out using either SigmaPlot 12.0 (Systat Software Inc.) built-in equations or our own ones.

Results: It turned out that, from the equations embedded in SigmaPlot, our experimental data can best be approximated using the equation of the 4-parameter log-normal distribution. However, herewith the "plateau" practically disappears on the growth curve. This problem can be avoided by using the equation developed by us, which is based on the much modified Verhulst-Pearl equation. As a result, the reliability of comparing the control and experimental curves is significantly increased. In particular, we managed to demonstrate a significant effect on the kinetics of cell growth and survival of the culture medium buffer capacity and the absence of such an effect in the case of exogenous 8-oxo-2'-deoxoguanosine, which was not obvious without the use of approximation. 